\title{
Design of parking management system for shared stroller based on RFID
}

\author{
Lina $\mathrm{Yu}^{1, *}$ \\ ${ }^{1}$ Computer Department, Shandong Xiehe University, Jinan, Shandong, China
}

Keywords: Shared stroller, RFID, Tag, Card reader.

\begin{abstract}
The development of information technology has led to the rapid development of sharing economy. Shared bike, Shared car and Shared stroller came into being. It not only brings convenience to citizens, but also has difficult management problems. This paper mainly proposes a solution to deal with the parking management of Shared stroller in shopping malls. The system is based on RFID technology. When the preset tag on the stroller is read successfully by the card reader,the stroller can be successfully locked and returned. Realize standardized management of Shared stroller in shopping malls.
\end{abstract}

\section{Research background}

The development of information technology makes our traditional life have new experience. Sharing economy is one of the new economic models, the emergence of Shared bike and car has brought convenience to people's life. Now many large shopping malls have also appeared Shared stroller. In order not to affect the environment of the shopping mall, facilitate the management of Shared stroller, and meet the needs of consumers, an intelligent parking system needs to be designed and developed.

Automatic identification technology is an important method and means for information and data automatic reading and input into computer. It is a comprehensive science and technology based on the development of computer technology and communication technology. In recent decades, automatic identification technology in the global scope has been rapid development.Initially formed a computer, optical, mechanical and electrical communication technology as one of the high-tech discipline.The disciplines include bar code technology, magnetic stripe card technology, optical character recognition, system integration, RFID, voice recognition and visual recognition

RFID technology is a kind of automatic identification technology. Realizes Non contact two - way data communication by radio frequency, useing radio frequency to read and write record media (electronic tag or radio frequency card),so as to achieve the purpose of identifying targets and data exchange. RFID non-contact automatic identification features can make the system more convenient and intelligent. Therefore, this paper designs a Shared stroller parking management system based on RFID.

\footnotetext{
*Corresponding author: 894907572@,163.com
} 


\section{System framework}

This system is based on RFID RFID technology. Install a reader in a specific area of the shopping mall and install a tag on each Shared stroller to complete non-contact two-way data transmission between reader and tag. As shown in Figure 1.

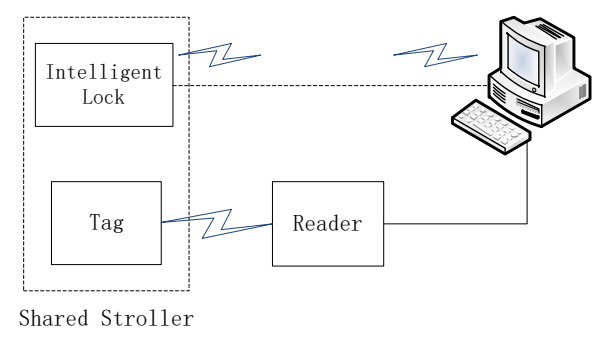

Fig. 1. Data transfer.

\subsection{Pick up}

Consumers use mobile APP to find Shared stroller, scan codes for stroller, and the system charges according to the time.

\subsection{Drop off}

When the stroller is returned, if the Shared stroller is parked in the reader reading area, the reader can succeed in reading the Shared stroller tag information. The host computer sends the lock signal to the Shared stroller through the network, at this time can lock the Shared stroller and complete the return steps. If the Shared stroller is not within the reading range of the reader, the host computer will not send a lock signal to the Shared stroller, then the consumer will not be able to successfully return the Shared stroller, and the timing charge will continue.

\subsection{Evaluation}

Each time the customer completes a normal return of the car, the service center will add one to the user's credit. When the credit is increased to a certain amount, users will be pushed red packets or coupons, to encourage regular use of Shared stroller.

\section{System software design}

This paper adopts NEWLab experimental platform to simulate the implementation, the system flow is shown in figure 2 . 


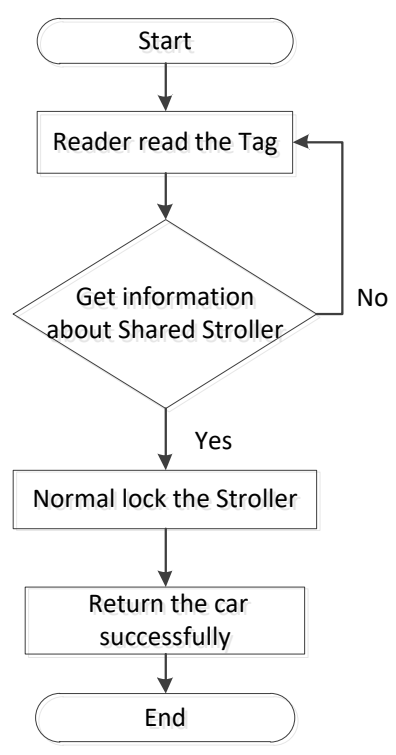

Fig. 2. System flowchart.

Introduce the main software of the system:

File.AppendAllText(String,String):

Function description: Opens a file, adds the specified string to the file, and then closes the file.If the file does not exist, this method can create a file and close it after writing the specified string to the file.

Namespace: System.IO

Attention: This system is with the help of File.AppendAllText(String,String)to save the registration information, and this method is a good solution to the problem of information coverage.

\section{The end}

This paper used NEWLab experimental platform to simulate the intelligent parking system of the Shared stroller. M3 core module, LF RFID module and motor module are selected for the system. Use the low frequency card registration card to simulate, When the Shared buggy in the parking system is parked in the designated area,the software interface shows "successfully locked the Shared stroller ". The host computer sends out a car lock signal, the motor rotates to lock the Shared stroller, return the car successfully. When the Shared stroller is far away from the reader and not in the reading range, the host computer will not send the lock signal, and the motor cannot lock the Shared stroller. The principle of the system is successfully simulated in this way.

Through the design of this system, the parking area is equivalent to setting up an electronic fence, which can improve the parking management problem of the Shared stroller in the mall, This system can not only facilitate the use of consumers, but also ensure the beauty of the mall.

\section{References}

1. Pei-wei W. Comparative analysis of rfid tag collision prevention algorithm. J. Internet of Things Technologies. 2017(04 )21-24. 
2. Longxu L. Countermeasures for regulating bike-sharing. J. Value engineering. 2017( 35) 205-6.

3. De G, Chunshuai Z, Yuanyuan Z, Lingfeng W and Weiyan T. Design of pileless spot parking system for Shared bicycles based on RFID technology. J. Information system engineering .2019(02)125-8.

4. Guangpei R, Yiwen L, Dongjiu Y and Chaojie C. Research on sustainable use strategy of buggy under the concept of sharing. J. Packaging engineering. 2018(39 )178-183.

5. Site F. Improvement of Shared bicycle parking mode -- design of Shared house. J. Journal of jishou university (social science edition). 2017,38(S2) 122-6.

6. Jingwei $\mathrm{W}$ and Zhiguang $\mathrm{Z}$. Research on green management mode of Shared bikes introduced by third-party organizations. J. Science and management .2019(06) $1-9$.

7. Linfeng L, Jinqiang L and Lian G. Gis-based site selection planning of urban Shared bicycle virtual site -- a case study of minjiang university campus. J. Smart city. 2019(05)4-8.

8. Rui N. Fragmented and integrated management of quasi-public goods supply in the context of sharing economy -- a case study of Shared bicycles. J.Contemporary finance and economics. 2019(10) 36-45. 